\title{
Atypical response with bone pseudoprogression in a patient receiving nivolumab for advanced cutaneous squamous cell carcinoma
}

\author{
Leandro J. C. Oliveira', Aline B. L. Gongora' ${ }^{1}$, Felipe G. Barbosa ${ }^{2}$, Carlos H. dos Anjos ${ }^{3}$ and Rodrigo R. Munhoz ${ }^{1,4^{*}}$ (D)
}

\begin{abstract}
Currently, there is no established standard of care for patients with metastatic CSCC. Based on the mechanisms of CSCC carcinogenesis has been postulated that these tumors may be amenable to PD-1/PD-L1 blockade. This case illustrates a patient with CSCC with nodal involvement and pulmonary metastases, refractory to two lines of platinum-based regimens and salvage surgery, for whom treatment with nivolumab was recommended. His clinical course was marked by an atypical pattern of response, with initial reduction of soft tissue/visceral lesions, yet development of new bone findings, followed by overall improvement in subsequent scans and sustained disease control upon treatment continuation.

The case of patient with metastatic CSCC, refractory, received immunotherapy and evolved with atypical pattern of response.
\end{abstract}

\section{Introduction}

Cutaneous SCC (CSCC) is among the most frequent malignancies worldwide and accounts for approximately $20 \%$ of all cutaneous neoplasms [1,2]. Although the incidence of CSCC has increased over the past decades, accurate estimates of the incidence and prevalence of CSCC may be biased by underreporting and misdiagnosis. A systematic review published in 2012 reunited 75 studies from different geographic regions. The highest incidence was observed in Australia (up to 1035 cases/100.000 personsyear), but elevated rates were also reported in the United States (up to 290 cases / 100.000 persons-year) and Europe (34.4 cases /100.000 persons-year for males and 15 /100.000 persons- years for females) [3].

Surgical excision remains the treatment of choice for the majority of the patients. Due to a pronounced sensitivity to radiation therapy, this treatment modality may be considered for individuals who are poor surgical

\footnotetext{
* Correspondence: rodrigo.rmunhoz@hsl.org.br

'Oncology Center - Hospital Sírio Libanês, Rua Dona Adma Jafet, 91. 2nd floor. Building A, São Paulo 01308-050, Brazil

${ }^{4}$ Oncology Service, Instituto do Câncer do Estado de São Paulo, Universidade de São Paulo, São Paulo, Brazil

Full list of author information is available at the end of the article
}

candidates, those with locally advanced disease or in situations in which excision followed reconstruction would be cosmetically/functionally unacceptable. Alternatively, radiation therapy with adjuvant intent is often indicated when tumor margins are positive, in the presence of perineural invasion or involvement of adjacent structures or in the setting of recurrent disease [4].

Besides an estimated risk of nodal involvement by CSCC of 3,7 - 5,4\%, distant metastases may develop in the lungs, liver, brain, bones and other cutaneous locations, leading to an ominous prognosis [5]. Currently, there is no established standard of care for patients with metastatic CSCC, and treatment decisions are largely based on extrapolations from regimens applicable to non-cutaneous SCC. Several agents have shown some activity in small, prospective trials or retrospective series, including cytotoxic chemotherapy (i.e cisplatin, 5-fluorouracil, bleomycin, methotrexate and doxorubicin), 13-cis-retinoic acid (13cRA) and interferon-2a [6]. In addition to standard chemotherapy, monoclonal antibodies against the epidermal growth factor receptor (EGFR) also have demonstrated activity in patients with CSCC. In a prospective study, cetuximab resulted in an overall disease control at 6 weeks 
of $69 \%$ and an objective response rate of $28 \%$. However, benefit is often short-lived. Also, it is important to note that a significant percentage of skin cancer patients are diagnosed at older ages with comorbidities which usually limit to the aapplicability of more intensive treatment regimens [7].

Programmed cell death receptor 1 (PD-1) is an inhibitory co-receptor expressed by T cells. Binding of PD-1 with its ligands (PD-L1/PD-L2) suppresses anti-tumor responses $[8,9]$. The development of monoclonal antibodies targeting the PD-1/PD-L1 axis, or immune checkpoint blockers, has dramatically changed the treatment of different types of malignancies, including squamous-cell carcinoma of the lung cancer and head and neck cancer, renal cell carcinoma [10-17], and a growing number of indications is being exhaustively investigated. Considering that chronic sun exposure, prior radiation, chronic inflammation and skin injury are major risk factors for CSCC, a significant benefit could emerge from strategies exploring immunecheckpoint blockade due to oncogenic mechanisms that involve pronounced DNA damage and an "inflamed" phenotype $[8,18]$.

We present a case of a patient developing an atypical pattern of response, marked by bone pseudoprogression during therapy with nivolumab for advanced CSCC, followed by sustained response.

\section{Case report}

A 72-year-old man presented with a history of multiple resections of CSCC located in his face, back and upper extremities. Almost 2 years subsequent to the latest excision, he first noted a palpable right axillary mass. A computed tomography $(\mathrm{CT})$ of the chest revealed a $10 \mathrm{~cm}$ right axillary nodal conglomerate, additional supraclavicular lymphadenopathies and pulmonary nodules concerning for metastatic disease. These findings were subsequently confirmed by an FDG-PET/CT, and a right axillary mass biopsy was consistent with moderately-differentiated CSCC. First-line therapy with cisplatin $75 \mathrm{mg} / \mathrm{m}^{2}$ combined with 5-fluorouracil $1000 \mathrm{mg} / \mathrm{m}^{2}$ days 1-4 was recommended, and accompanied by significant gastrointestinal toxicity (diarrhea/mucositis). Re-staging scans revealed a reduction in size of the previously appreciable lesions. A salvage right axillary lymphadenectomy was attempted at an outside institution, revealing involvement by CSCC of 15 out of 16 resected lymph nodes. Early disease progression led to the indication of second-line therapy with carboplatin AUC 5 and paclitaxel $175 \mathrm{mg} / \mathrm{m}^{2}$, given every 21 days. Following cycle 6 , repeat imaging showed disease progression in the lungs and multiple lymphadenopathies: right axillary, cervical, retropectoral and mediastinal. Of note, no signs of bone involvement were noted, despite degenerative findings and a previously described fracture attributed to osteoporosis.
At that point, the patient transitioned his care to our service. Following extensive debate at a multidisciplinary tumor board, decision was made to proceed with third-line nivolumab. After a thorough discussion and clarification regarding the off-label use of the anti-PD-1 agent, the patient received the first dose of nivolumab $3 \mathrm{mg} / \mathrm{kg}$ given intravenously every 14 days. Except for non-limiting fatigue, the patient had a remarkable tolerance to nivolumab, accompanied by early clinical response (reduction of right axillary/retropectoral mass). Re-staging PET-CT performed after 3 months of therapy revealed reduction in size and resolution of the metabolic activity of right axillary, cervical, retropectoral and mediastinal lymphadenopathies; pulmonary nodules were no longer appreciable. However, new areas of hypermetabolism were noted in C7, T1, T6 and L1 vertebral bodies, along with new lytic lesions, as well as increased metabolic activity in $\mathrm{T} 3, \mathrm{~T} 4$ and $\mathrm{T} 7$ pedicles (Figs 1 and 2).

Due to the limited treatment options in this setting and remarkable response of visceral/nodal lesions, and based on a shared decision, continued treatment with nivolumab followed by short-interval repeat (6-8 weeks) scans was recommended. The patient decided to proceed with his treatment locally, at an outside facility, and returned for a reevaluation almost 5 months later, still receiving nivolumab and with no new adverse events. A new PET-CT showed metabolic response in the previously identified bone lesions, now described as sclerotic, and sustained control of pulmonary nodules and lymphadenopathies (Figs 1 and 2). Following more than 12 of nivolumab therapy, the patient continues to endure clinical benefit, with prolonged disease control and good tolerance.

\section{Discussion}

This case illustrates a patient with CSCC with multifocal nodal involvement and pulmonary metastases, refractory to two lines of platinum-based combination regimens and salvage surgery, for whom treatment with nivolumab was recommended. His clinical course was marked by an atypical pattern of response, with initial reduction of soft tissue/visceral lesions, yet development of new bone findings, followed by overall improvement in subsequent scans and sustained disease control upon treatment continuation. In addition to the remarkable radiologic findings, this case highlights the potential for the use of immune-checkpoint blockade for patients with advanced CSCC.

In Brazil, where the patient was treated, non-melanoma skin cancer is among the most frequently diagnosed neoplasms, corresponding to $30 \%$ of all malignant tumors in this country [19]. There is an unmet need for evidence to guide treatment decisions in patients with advanced CSCC. 


\section{$31 / 10 / 2016$}

A

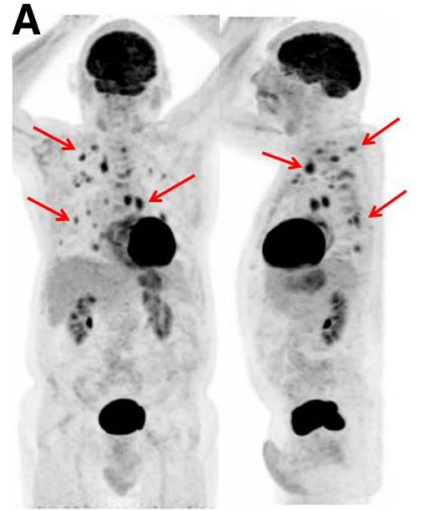

$10 / 02 / 2017$

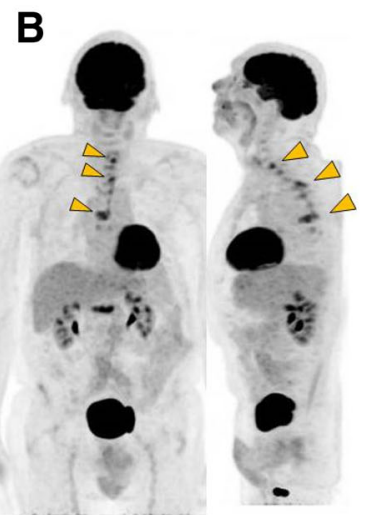

$21 / 07 / 2017$

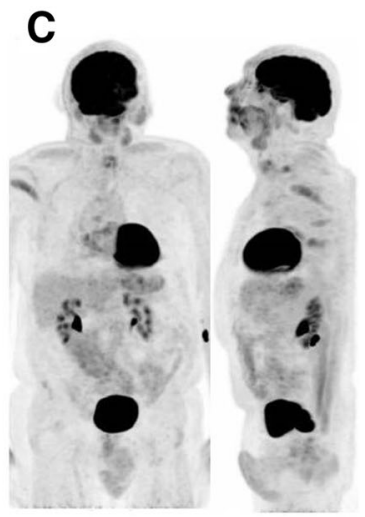

Fig. 1 Evolution of pulmonary, mediastinal and vertebral lesions seen in ${ }^{18} \mathrm{~F}-\mathrm{FDG}$ PET/CT's MIP images during treatment with anti-PD1. MIP images (front and lateral view) from different ${ }^{18} \mathrm{~F}-\mathrm{FDG}$ PET/CT's. a Multiple FDG avid lesions in the lungs, mediastinum and thoracic vertebral bodies. b Metabolic resolution of the metastatic lung and mediastinal lesions, however there is increased FDG uptake in previous thoracic bone lesions associated with new hypermetabolic bone lesion (arrowhead). c Metabolic resolution of most thoracic bone lesions, consistent with partial metabolic response (PMR)

\section{$31 / 10 / 2016$}
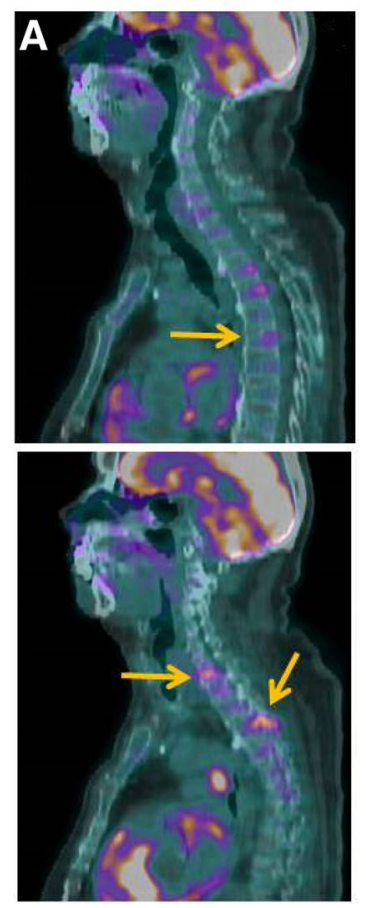

$10 / 02 / 2017$
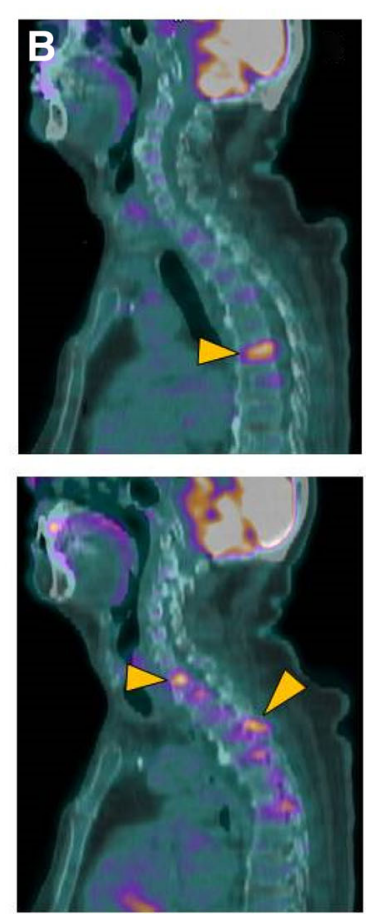

$21 / 07 / 2017$
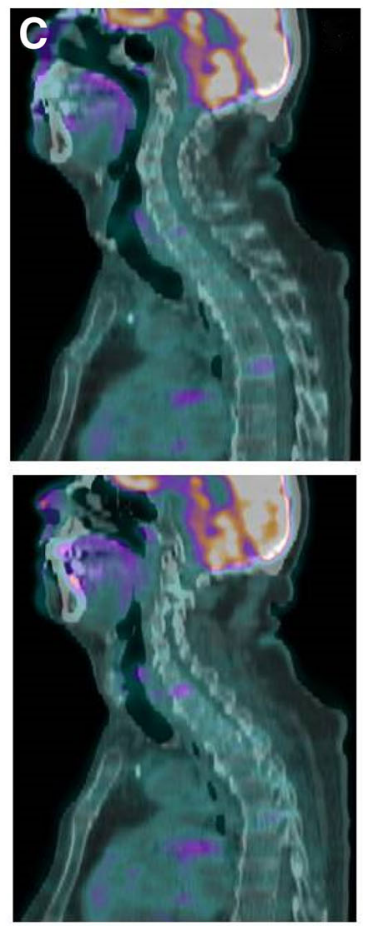

Fig. 2 Bone Pseudoprogression after anti-PD1 therapy seen in ${ }^{18} \mathrm{~F}-\mathrm{FDG}$ PET/CT's. Sagital fused images from different ${ }^{18} \mathrm{~F}-\mathrm{FDG}$ PET/CT's. a Multiple focal FDG uptake in different thoracic vertebrae (T1, T3 and T7) without tomographic focal lesion (arrows), corresponding to bone metastasis. $\mathbf{b}$ Same corresponding thoracic bone lesions with significant increased uptake (arrowsheads), consistent with metabolic progression disease. c Metabolic resolution of most thoracic bone lesions, consistent with partial metabolic response (PMR) 
Based on the mechanisms of CSCC carcinogenesis and potential markers of immunogenicity [8], it has been postulated that these tumors may be amenable to PD-1/ PD-L1 blockade. In a phase 1 study of REGN2810/Cemiplimab, an anti-PD-1 antibody, good tolerability and pronounced activity was documented in 26 patients with metastatic or locally advanced CSCC, with overall response and disease control rates of 46.2 and $69.2 \%$, respectively [18]. Of note, $81 \%$ of pre-treatment tumor samples expressed PD-L1 [18]. In a retrospective cases series of patients with CSCC treated with anti-PD-1 agents, five of six patients (83\%) achieved a clinical response, with 1 complete and 4 partial responses. The median duration of PD-1 inhibitor exposure was 8 months, and the longest PFS interval was 21 months [20]. More recently, the combined analysis of data from the expansion cohort of a phase I trial $(N=26)$ and from a phase II study $(N=59)$, totaling 85 CSCC patients treated with cemiplimab, suggested objective response rates of $50 \%$ in the expansion cohort and $47 \%$ in the phase II cohort, with responses exceeding 6 months in $57 \%$ of the responders. Of note, treatment was well tolerated, and only $7 \%$ of the patients discontinued the treatment due to adverse events [21].

This case also illustrates one the challenges involved in the efficacy assessment is patients treated with immunotherapy. Responses to immune-checkpoint blockade may be considerably diverse from those observed with either cytotoxic chemotherapy or targeted-therapy. Due to mechanisms not fully characterized, an initial growth in tumor size or appearance of new lesions (pseudoprogression) or late tumor regression may occur, followed by delayed, and occasionally durable responses [22-24]. The rate of these so called atypical patterns of response may vary according to the type of monoclonal antibody, disease and response evaluation criteria applied; a recently published systematic review reported an incidence of atypical responses of approximately $6 \%$ in patients with distinct solid tumors treated with antiPD-1 agents [23]. Pseudoprogression in spinal metastases has been described in other cancer types treated with immunotherapy, including a case report of a patient with melanoma treated with immune checkpoint blockade and radiation therapy [25]. Due to such atypical patterns of response, innovative response assessment criteria have been suggested, but not yet fully implemented, including the immune-related response criteria (irRC) and the modified Response Evaluation Criteria in Solid Tumors (iRECIST) [21, 26].

To the best of our knowledge, this is the first report of this pattern of pseudoprogression (new bone lesions) in a patient receiving nivolumab for CSCC and reinforces the need for individualized response criteria and a judicious assessment in order to avoid premature discontinuation of treatment with potential benefits. It also highlights the promising activity demonstrated by immune-checkpoint blockade in patients with CSCC: although prospective, controlled trials are warranted, the use of immunotherapy-based approaches may become the standard of care in the near future for patients affected by this challenging disease.

\section{Acknowledgements \\ Not applicable.}

Funding

No funding received.

\section{Availability of data and materials}

The data that support the findings of this study are available from the corresponding author upon reasonable request.

\section{Authors' contributions}

LCO and ABLG described the case and wrote the initial article. RRM was the patient's personal doctor and was a reviewer of the article, together with CHA. FGB analysed, selected and wrote the images subtitles. All authors read and approved the final manuscript.

Ethics approval and consent to participate Not applicable.

\section{Competing interests}

RRM - Research involvement: BMS/Merck Serono/Novartis/Roche; Honoraria: BMS/MSD/Roche/Novartis; Travel grants: BMS/MSD/Roche/Teva/Novartis; Advisory role: Merck Serono/MSD/Roche. Additional authors declare that they have no competing interests.

\section{Publisher's Note}

Springer Nature remains neutral with regard to jurisdictional claims in published maps and institutional affiliations.

\section{Author details}

'Oncology Center - Hospital Sírio Libanês, Rua Dona Adma Jafet, 91. 2nd floor. Building A, São Paulo 01308-050, Brazil. ${ }^{2}$ Radiology and Nuclear Medicine Service - Hospital Sírio Libanês, São Paulo, Brazil. ${ }^{3}$ Oncology Center - Hospital Sírio Libanês, Brasília, Brazil. ${ }^{4}$ Oncology Service, Instituto do Câncer do Estado de São Paulo, Universidade de São Paulo, São Paulo, Brazil.

Received: 6 September 2018 Accepted: 5 November 2018

Published online: 27 November 2018

\section{References}

1. Apalla Z, Lallas A, Sotiriou E, et al. Epidemiological trends in skin cancer. Dermatol Pract Concept. 2017;7(2):1-6.

2. Rogers HW, Weinstock MA, Feldman SR, et al. Incidence estimate of nonmelanoma skin Cancer (keratinocyte carcinomas) in the US population, 2012. JAMA Dermatol. 2015;151(10):1081-6.

3. Lomas A, Bee-Leonardi J, Bath-Hextall F. A systematic review of worldwide incidence of non-melanoma skin cancer. Br J Dermatol. 2012;166:1069-80.

4. Parikh SA, Patel VA, Raatner D. Advances in the management of cutaneous squamous cell carcinoma. F1000Prime Rep. 2014;6(70).

5. Murad A, Ratner D. Cutaneous Squamous-Cell Carcinoma. N Engl J Med. 2001;344(13):975-83

6. Stratigos A, Garbe C, Lebbe C, et al. Diagnosis and treatment of invasive squamous cell carcinoma of the skin: European consensus-based interdisciplinary guideline. Eur J Cancer. 2015;51(14):1989-2007.

7. Maubec E, Petrow P, Scheer-Senyarich I, et al. Phase II study of cetuximab as first-line single-drug therapy in patients with unresectable squamous cell carcinoma of the skin. J Clin Oncol. 2011;29(25):3419.

8. Stevenson ML, Wang CQF, Abikhair M, et al. Expression of programmed cell death ligand in cutaneous squamous cell carcinoma and treatment of locally advanced disease with Pembrolizumab. JAMA Dermatology. 2017; 153(4):299-303. 
9. Safarty M, Moore A, Dudnik E, et al. Not only for melanoma. Subcutaneous pseudoprogression in lung squamous-cell carcinoma treated with nivolumabe - A case report. Medicine. 2017;96(4):1-2.

10. Topalian SL, Sznol M, McDermott DF, et al. Survival, durable tumor remission, and long-term safety in patients with advanced melanoma receiving nivolumab. J Clin Oncol. 2014;32(10):1020-31.

11. Weber JS, D'Angelo SP, Minor D, et al. Nivolumab versus chemotherapy in patients with advanced melanoma who progressed after anti-CTLA4 treatment (CheckMate037): a randomised, controlled, open-label, phase 3 trial. Lancet Oncol. 2015;16:375-84.

12. Robert C, Long GV, Brady B, et al. Nivolumab in previously untreated melanoma without a BRAF mutation. N Engl J Med. 2014;372(4):320-30.

13. Rizvi NA, Mazieres J, Planchard D, et al. Activity and safety of nivolumab, an anti-PD1 immune checkpoint inhibitor, for patients with advanced, refractory squamous non-small-cell lung cancer (CheckMate063): a phase 2, single-arm trial. Lancet Oncol. 2015;16:257-65.

14. Brahmer J, Reckamp KL, Baas $\mathrm{P}$, et al. Nivolumab versus docetaxel in advanced squamous-cell non-small-cell lung Cancer. N Engl J Med. 2015; 373:123-35.

15. Borghaei H, Paz-Ares L, Horn L, et al. Nivolumab versus docetaxel in advanced nonsquamous non-small-cell lung cancer. N Engl J Med. 2015; 373:1627-39.

16. Motzer RJ, Escudier B, McDermott DF, et al. Nivolumab versus everolimus in advanced renal-cell carcinoma. N Engl J Med. 2015;373:1803-13.

17. Ferris RL, Blumenschein G Jr, Fayette J, et al. Nivolumab for recurrent squamous-cell carcinoma of the head and neck. N Engl J Med. 1856-1867; 375(19):2016.

18. Papadopoulos KP, Owonikoko TK, Johnson ML, et al. REGN2810: A fully human anti-PD-1 monoclonal antibody, for patients with unresectable locally advanced or metastatic cutaneous squamous cell carcinoma (CSCC) - Initial safety and efficacy from expansion cohorts (ECS) of phase I study. Journal of Clinical Oncology. 2017;35(15suppl):9503.

19. Estimate 2016: Cancer Incidence in Brazil/ Instituto Nacional de Câncer José Alencar Gomes da Silva - Rio de Janeiro. INCA 52-53, 2015.

20. Tran DC, Colevas AD, Chang AL. Follow-up on programmed cell death inhibitor for cutaneous squamous cell carcinoma. JAMA Dermatol. 2017 Jan 1;153(1):92-4.

21. Migden MR, Rischin CD, Schmults A, et al. PD-1 blockade with cemiplimab in advanced cutaneous squamous-cell carcinoma. N Engl J Med. 2018; 379(4):341-51.

22. Wolchok JD, Hoos A, O'Day S, et al. Guidelines for the evaluation of immune therapy activity in solid tumors: immune-related response criteria. Clin Cancer Res. 2009;15(23):7412-20.

23. Queirolo P, Spagnolo F, et al. Atypical responses in patients with advanced melanoma, lung cancer, renal-cell carcinoma and other solid tumors treated with anti-PD-1 drugs: a systematic review. Cancer Treat Rev. 2017:59:71-8.

24. Hodi FS, Hwu WJ, Kefford R, et al. Evaluation of immune-related response criteria and RECIST v1.1 in patients with advanced melanoma treated with pembrolizumab. J Clin Oncol. 2016;34:1510-7.

25. Jhawar SR, Silk AW, Goyal S. Pseudoprogression of a spinal metastasis after stereotactic ablative body radiation therapy and immune checkpoint therapy. Practical Radiation Oncology. 2017;7(2):109-12.

26. Seymour L, Bogaerts J, Perrone A, et al. iRECIST: guidelines for response criteria for use in trials testing immunotherapeutics. Lancet Oncol. 2017; 18(3):143-52.

Ready to submit your research? Choose BMC and benefit from:

- fast, convenient online submission

- thorough peer review by experienced researchers in your field

- rapid publication on acceptance

- support for research data, including large and complex data types

- gold Open Access which fosters wider collaboration and increased citations

- maximum visibility for your research: over $100 \mathrm{M}$ website views per year

At BMC, research is always in progress.

Learn more biomedcentral.com/submissions 